\title{
Pacific
}

Journal of

Mathematics

\section{UNIQUENESS THEOREMS FOR CR AND CONFORMAL MAPPINGS}

\author{
YounG-Jun CHOI AND JAE-CHEON JOO
}




\title{
UNIQUENESS THEOREMS FOR CR AND CONFORMAL MAPPINGS
}

\author{
YounG-Jun CHOI AND JAE-CHEON JOO
}

\begin{abstract}
We provide a uniqueness theorem for $\mathrm{CR}$ and conformal mappings that generate compact sequences of iteration.
\end{abstract}

\section{Introduction}

The primary aim of this paper is to prove a version of uniqueness theorem for $\mathrm{CR}$ and conformal mappings.

Let $M$ be a $C^{\infty}$-smooth manifold and let $\mathscr{C}$ be a class of smooth mappings from $M$ into itself containing the identity map. We say that the pair $(M, \mathscr{C})$ satisfies the Cartan uniqueness property (or simply Cartan uniqueness) at $p \in M$ if an element $f \in \mathscr{C}$ coincides with the identity map whenever $f(p)=p, d f_{p}=\operatorname{Id}_{T_{p} M}$ and

$$
\left\{f^{k}: k \in \mathbb{Z}_{+}\right\}
$$

is compact, where $\operatorname{Id}_{T_{p} M}$ is the identity transform of tangent space $T_{p} M$ of $M$ at $p, \mathbb{Z}_{+}$is the set of nonnegative integers, and $f^{k}$ is the $k$-time composition of $f$, namely,

$$
f^{k}=\underbrace{f \circ \cdots \circ f}_{k} .
$$

In this definition, the compactness of a subclass $\mathscr{C}^{\prime}$ of $\mathscr{C}$ means that every sequence in $\mathscr{C}^{\prime}$ contains a subsequence that is uniformly Cauchy on every compact subset of $M$. (In other words, $\mathscr{C}^{\prime}$ is relatively compact with respect to the compact-open topology.) One of the most important examples of this property in complex analysis is the uniqueness theorem of $\mathrm{H}$. Cartan.

Theorem 1.1 (The Cartan uniqueness theorem). Let $\Omega$ be a domain in $\mathbb{C}^{n}$ and let H be the class of holomorphic mappings from $\Omega$ into itself. Then $(\Omega, \mathcal{H})$ satisfies the Cartan uniqueness property at every point of $\Omega$. In particular, if $\Omega$ is bounded, then $f \in \mathcal{H}$ coincides with the identity map if $f(p)=p$ and $d f_{p}=\operatorname{Id}_{T_{p} \Omega}$ for some $p \in \Omega$, since the sequence $\left\{f^{k}: k \in \mathbb{Z}_{+}\right\}$is automatically compact by the Montel theorem.

MSC2010: 32V10, 32V40, 53A30.

Keywords: Cartan uniqueness, CR mappings, conformal mappings. 
It is not trivial to determine under what conditions a similar uniqueness theorem holds if the reference point $p$ lies on the domain boundary. For biholomorphic mappings we have the following theorems:

Theorem 1.2 [Krantz 1987]. Suppose that $\Omega$ is a strongly pseudoconvex domain that is not biholomorphic to the ball. Let $f: \Omega \rightarrow \Omega$ be a biholomorphic mapping and let $p \in \partial \Omega$. If $f(z)=z+o(|z-p|)$, then $f \equiv \mathrm{Id}$.

Theorem 1.3 [Huang 1993]. Suppose that $\Omega$ is a bounded pseudoconvex domain satisfying Condition $R$. Let $f: \Omega \rightarrow \Omega$ be a biholomorphic map and let $p \in \partial \Omega$. If $f(z)=z+o(|z-p|)$ and $\left\{f^{k}: k \in \mathbb{Z}\right\}$ is compact, then $f \equiv \mathrm{Id}$.

A crucial part in the proof of the Cartan uniqueness theorem are the Cauchy estimates for holomorphic mappings, which are consequences of the ellipticity of $\bar{\partial}$. In contrast, the operator $\bar{\partial}$ does not enjoy ellipticity or even subellipticity on the boundary of a domain. Huang [1993] exploited Bell's theorem [1987] on the boundary behavior of biholomorphic mappings in the $C^{\infty}$ smooth sense, which is obtained from an analysis of the transformation formula of the Bergman kernel function.

In this paper, we prove a $\mathrm{CR}$ version of the uniqueness theorem:

Theorem 1.4 (CR case). Let $M$ be either a real hypersurface in $\mathbb{C}^{n+1}$ that does not contain any analytic hypersurface or a compact real hypersurface that bounds a domain. Let $\mathscr{H}_{b}$ be the class of all CR mappings from $M$ into itself. Then $\left(M, \mathscr{H}_{b}\right)$ satisfies the Cartan uniqueness property at every strongly pseudoconvex point.

The main interest of this theorem is that we assume neither a global type condition on $M$ nor global injectivity of the mappings in the class $\mathscr{H}_{b}$. Therefore, we may regard this theorem as a generalization of Theorem 1.2 in the CR case. The proof of Theorem 1.4 is based on the method of derivative estimates of CR diffeomorphisms by the local solvability of the CR Yamabe equation, which was mainly developed in [Schoen 1995; Fischer-Colbrie and Schoen 1980].

By considering the conformal Yamabe equation instead of the CR Yamabe equation, we can have a conformal version of Theorem 1.4. For given two Riemannian manifolds $(M, g)$ and $(N, h)$, a diffeomorphism $f$ from $M$ to $N$ is said to be conformal if $f^{*} h=u g$ for some positive function $u$ on $M$. We define a little bit wider class of mappings as follows.

Definition 1.5. Let $(M, g)$ and $(N, h)$ be Riemannian manifolds. A smooth map $f: M \rightarrow N$ is said to be semiconformal if $f^{*} h=\lambda g$ for some smooth function $\lambda$. In this definition, we assume neither that $f$ is $1-1$ nor $\lambda>0$.

Theorem 1.6 (Conformal case). Let $(M, g)$ be a Riemannian manifold of dimension $n>2$, and let 9 be the class of all semiconformal mappings from $M$ into itself. Then $(M, \mathscr{Y})$ satisfies the Cartan uniqueness property at every point of $M$. 
We present the proof of Theorem 1.6 in Section 2 and then prove Theorem 1.4 in Section 3. Each section contains fundamental definitions of corresponding geometric objects - Yamabe equation, CR and pseudohermitian structures, CR mappings and so on.

\section{Proof of Theorem 1.6}

We start this section by recalling the Yamabe equation and problem. Let $(M, g)$ be a Riemannian manifold of real dimension $n \geq 3$. Let $\tilde{g}=e^{2 f} g$ be a conformal change of a Riemannian metric $g$, where $f$ is smooth real-valued function on $M$. If we denote by $S$ and $\widetilde{S}$ the scalar curvatures of $g$ and $\tilde{g}$, respectively, it turns out that they satisfy the transformation law

$$
\widetilde{S}=e^{-2 f}\left(S-2(n-1) \Delta_{g} f-(n-1)(n-2)\left|\nabla_{g} f\right|^{2}\right),
$$

where $\Delta_{g} f$ denotes the Laplacian — the trace of the second covariant derivative of $f$ and $\nabla_{g} f$ its covariant derivative for the metric $g$. Let $\phi$ be the positive function satisfying $e^{2 f}=\phi^{p_{n}-2}$, where $p_{n}=2 n /(n-2)$. Then the equation above turns into the following nonlinear equation for $\phi$ :

$$
-a_{n} \Delta_{g} \phi+S \phi=\widetilde{S} \phi^{p_{n}-1},
$$

where $a_{n}=4(n-1) /(n-2)$. This is called the Yamabe equation and the linear operator $L_{g}=-a_{n} \Delta_{g}+S$ is called the conformal Laplacian for $g$. When we mention the Yamabe problem, we mean the problem of finding a positive solution $\phi$ of (2-1) that makes $\widetilde{S}$ constant. This problem was first introduced in [Yamabe 1960], and its solvability has been intensively investigated there and elsewhere [Trudinger 1968; Aubin 1976a; 1976b; Schoen 1984; 1995].

For our purposes, a local scalar flattening argument is needed rather than the global solvability of the Yamabe problem:

Theorem 2.1 [Fischer-Colbrie and Schoen 1980]. Let $(M, g)$ be a Riemannian manifold and let $Q$ be a smooth function on $M$. For $x \in M$ and $R>0$, we denote by $B_{R}(x)$ the geodesic ball centered at $x$ of radius $R$. If the minimum eigenvalue

$$
\lambda\left(B_{R}(x)\right)=\inf \left\{\int_{B_{R}(x)}\left(|\nabla f|^{2}+Q f^{2}\right) d V: \operatorname{Support}(f) \subset B_{R}(x), \int_{B_{R}(x)} f^{2}=1\right\}
$$

of $\Delta_{g}-Q$ on $B_{R}(x)$ is positive, then there exists a positive function $\phi$ on $M$ such that

$$
\left(\Delta_{g}-Q\right) \phi=0
$$

on $B_{R}(x)$. 
Let us return back to the situation of Theorem 1.6. Let $p$ be a fixed point of $M$ and let $f: M \rightarrow M$ be a semiconformal map satisfying that $f(p)=p, d f_{p}=\operatorname{Id}_{T_{p}} M$ and that $\left\{f^{k}: k \in \mathbb{Z}_{+}\right\}$is compact. Then we can choose a neighborhood $U=B_{R}(p)$ of $p$ such that

(i) $f$ is one-to-one on $U$.

Choosing $R$ small enough, we may also assume that the minimum eigenvalue of $-L_{g}$ is positive on $U$ by the Poincaré inequality (see [Gilbarg and Trudinger 1983], for example), and that

$$
\|u\|_{q} \leq C R\|\nabla u\|_{q}
$$

for every $u \in C_{0}^{\infty}(U)$ and $1 \leq q<\infty$, where $C$ is a constant depending only on the dimension $n$. Therefore, there exists a positive function $\phi$ on $M$ such that $L_{g} \phi=0$ on $U$ by Theorem 2.1. Replacing the metric $g$ by $\phi^{4 /(n-2)} g$, then we may assume that

(ii) the metric $g$ is scalar flat on $U$

by the Yamabe equation (2-1).

Thanks to the assumption that $\left\{f^{k}: k \in \mathbb{Z}_{+}\right\}$is compact, there exists a neighborhood $V$ of $p$ that is relatively compact in $U$, such that $f^{k}(V) \subset U$ for every $k=1,2, \ldots$ By (i), $f^{k}$ is a conformal transformation from $V$ to $f^{k}(V) \subset U$. Therefore, there exists a positive function $u_{k}$ on $V$ such that

$$
\left(f^{k}\right)^{*} g=\left(u_{k}\right)^{4 /(n-2)} g
$$

for every $k=1,2, \ldots$ We denote $u_{1}$ by $u$.

Since $g$ and $\left(f^{k}\right)^{*} g$ are scalar flat on $V, u_{k}$ satisfies the homogeneous Yamabe equation

$$
\Delta_{g} u_{k}=0
$$

on $V$ by (2-1).

Since $f^{k}$ is a one-to-one map from $V$ into $U$, it follows that

$$
\int_{V} u_{k}^{2 n /(n-2)} d V_{g}=\operatorname{Vol}_{\left(f^{k}\right)^{*} g} V=\operatorname{Vol}_{g} f^{k}(V) \leq \operatorname{Vol}_{g} U<\infty .
$$

By the elliptic mean value inequality, there exists $C>0$ such that $u_{k}<C$ for every $k$ on a neighborhood $V^{\prime}$ of $p$ that is relatively compact in $V$.

Let $V^{\prime \prime}$ be a neighborhood of $p$ that is relatively compact in $V^{\prime}$. By the elliptic estimate for $\Delta_{g}$ [Gilbarg and Trudinger 1983], there is a $C_{j}$ independent of $k$ such that

$$
\left\|u_{k}\right\|_{C^{j}\left(V^{\prime \prime}\right)}<C_{j}
$$


Note that

$$
u_{k}(x)=u\left(f^{k-1}(x)\right) \cdots u(f(x)) \cdot u(x)
$$

for every positive integer $k$. Let $x=\left(x^{1}, \ldots, x^{n}\right)$ be local coordinates on $V$ centered at $p$ and let

$$
u(x)=1+h_{j}(x)+O\left(|x|^{j+1}\right),
$$

where $h_{j}$ is a $j$-th degree homogeneous polynomial. Since $f(x)=x+o(|x|)$ by the hypothesis, we see that

$$
u_{k}(x)=1+k h_{j}(x)+O\left(|x|^{j+1}\right)
$$

from (2-3). Therefore, if $h_{j}$ does not vanish, then the $j$-th order differential of $u_{k}$ at $p=0$ diverges as $k \rightarrow \infty$, which contradicts the inequality (2-2). This means that $h_{j}$ vanishes identically on $V^{\prime \prime}$, hence $v=u-1$ vanishes at $p$ up to infinite order. By the unique continuation principle [Garofalo and Lin 1987; Kazdan 1988], we have $v=0$ on $V^{\prime \prime}$, namely, $u=1$ on $V^{\prime \prime}$. This implies that $f$ is an isometry on $V^{\prime \prime}$. Therefore, $f$ coincides with the identity map on $V^{\prime \prime}$, since every local geodesic passing through $p$ should be preserved by $f$.

Let $F=\{x \in M: f(x)=x\}$ and let $F^{\circ}$ the interior of $F$. Since $V^{\prime \prime} \subset F^{\circ}$, we see that $F^{\circ}$ is nonempty. If $x_{0}$ is a limit point of $F^{\circ}$, then obviously $f$ satisfies that $f\left(x_{0}\right)=x_{0}$ and $d f_{x_{0}}=\operatorname{Id}_{T_{x_{0}} M}$. Repeating all the arguments above, we conclude that $x_{0} \in F^{\circ}$. Thus $F^{\circ}$ is closed. This completes the proof of Theorem 1.6.

Corollary 2.2. Let $f$ be a conformal transformation of $M$ such that $f(p)=p$ and $d f_{p}=\operatorname{Id}_{T_{p} M}$. Then either $f \equiv \operatorname{Id}$ or $M$ is conformally equivalent to the unit sphere $S^{n}$ and $f$ can be transformed into the conformal transformation $\phi_{a}$ of $S^{n}$ that fixes $p_{0}=(1,0, \ldots, 0) \in \mathbb{R}^{n+1}$ for some $a=\left(a_{1}, \ldots, a_{n}\right) \in \mathbb{R}^{n}$, where $\phi_{a}=\Phi_{a} /\left|\Phi_{a}\right|$ and $\Phi_{a}$ is the affine transformation of $\mathbb{R}^{n+1}$ defined by

$$
\begin{aligned}
& y_{0}=\frac{|a|^{2}}{4}+\left(1-\frac{|a|^{2}}{4}\right) x^{0}+\frac{1}{\sqrt{2}} \sum_{j=1}^{n} a_{j} x^{j}, \\
& y^{j}=x^{j}+\frac{a_{j}}{\sqrt{2}}\left(1-x^{0}\right)
\end{aligned}
$$

for $j=1, \ldots, n$.

Proof. By Theorem 1.6, we may assume that $\left\{f^{k}: k \in \mathbb{Z}\right\}$ is noncompact if $f$ is not the identity transform. Then Schoen's theorem [1995] implies that $M$ is conformally equivalent to either $\mathbb{R}^{n}$ or $S^{n}$. Since $\mathbb{R}^{n}$ is conformally equivalent to $S^{n} \backslash\left\{p_{\infty}=\right.$ $(-1,0, \ldots, 0)\}$, we may also assume that $f$ is a conformal transformation of $S^{n}$ fixing $p_{0}=(1,0, \ldots, 0)$. By Obata's theorem [1970], either $f$ has two fixed points or it has only one fixed point. Moreover, if $f$ has two fixed points, then each fixed 
point is a contracting fixed point of $f$ or $f^{-1}$. This contradicts the hypothesis that $d f_{p_{0}}=\operatorname{Id}_{T_{p_{0}} M}$. In particular, $M$ is conformally equivalent to $S^{n}$, since if $M$ were conformally equivalent to $\mathbb{R}^{n}$, then $f$ should fix two points $p_{0}$ and $p_{\infty}$ as a conformal transform of $S^{n}$. If $f$ has only one fixed point $p_{0}$ and $d f_{p_{0}}=\mathrm{Id}$, then $f=\Phi_{a} /\left|\Phi_{a}\right|$ for some $a \in \mathbb{R}^{n}$ by the algebraic characterization of the conformal transformation group of $S^{n}$ via projectivization. See [Obata 1970] for more details.

\section{Proof of Theorem 1.4}

We first review some definitions related to CR manifolds and CR mappings.

Let $M$ be a smooth manifold of real dimension $2 n+1$ and let $H$ be a subbundle of $T M$ with fiber dimension $2 n$. Let $J$ be a smooth section of the endomorphism bundle of $H$ satisfying $J^{2}=-\operatorname{Id}_{H}$. Then the triple $(M, H, J)$ is called a $C R$ manifold if it satisfies the integrability condition

$$
\left[\Gamma\left(H_{1,0}\right), \Gamma\left(H_{1,0}\right)\right] \subset \Gamma\left(H_{1,0}\right),
$$

where $H_{1,0}$ is the subbundle of $\mathbb{C} \otimes H$ on which $J=i$, and $\Gamma\left(H_{1,0}\right)$ is the space of smooth sections of $H_{1,0}$. A typical example of a $\mathrm{CR}$ manifold is a real hypersurface of a complex manifold. Let $(M, H, J)$ and $\left(M^{\prime}, H^{\prime}, J^{\prime}\right)$ be two CR manifolds. A smooth map $f: M \rightarrow M^{\prime}$ is called a $C R$ mapping if $d f\left(H_{1,0}\right) \subset H^{\prime}{ }_{1,0}$. For a CR manifold $(M, H, J)$, let $\theta$ be a nonvanishing real 1-form on $M$ that vanishes on $H$. The Levi form $L_{\theta}$ is the symmetric bilinear form on $H$ defined by

$$
\left(L_{\theta}\right)_{x}(X, Y)=d \theta_{x}(X, J Y)
$$

for every $x \in M$ and $X, Y \in H_{x}$.

A CR manifold $(M, H, J)$ is said to be strongly pseudoconvex if the Levi form $L_{\theta}$ for some $\theta$ is positive definite on $H$. In this case, the 1 -form becomes a contact form. The quadruple $(M, H, J, \theta)$ is called a pseudohermitian manifold; see [Webster 1978]. We abbreviate this by $(M, \theta)$.

For a pseudohermitian manifold $(M, \theta)$, let $\xi$ be the vector field on $M$ defined by $\theta(\xi)=1$ and $\xi\lrcorner d \theta=0$. Then for every $x \in M, T_{x} M=\left[\xi_{x}\right] \oplus H_{x}$, where $\left[\xi_{x}\right]$ denotes the space generated by $\xi_{x}$. This decomposition defines a natural projection $\pi: T_{x} M \rightarrow H_{x}$. We define a Riemannian metric $g$ on $M$ by

$$
g_{x}(X, Y)=\theta_{x}(X) \theta_{x}(Y)+\left(L_{\theta}\right)_{x}(\pi(X), \pi(Y))
$$

for every $X, Y \in T_{x} M$. The Tanaka-Webster connection $\nabla$ on $(M, \theta)$ is an affine connection for which $g, V$ and $J$ are parallel. This connection is determined uniquely under suitable conditions on the torsion tensor. See [Tanaka 1975; Webster 1978]. By differentiating the Tanaka-Webster connection form, the pseudohermitian 
curvature tensor can be defined. The trace of this tensor is the Ricci tensor, and the trace of the Ricci tensor is the Webster scalar curvature $R_{\theta}$. Obviously, the Webster scalar curvature depends the choice of the contact form $\theta$.

Let $\tilde{\theta}=u^{2 / n} \theta$ be another choice of contact form, where $u$ is a positive smooth function, and let $\widetilde{R}$ and $R$ be the Webster scalar curvatures for $\tilde{\theta}$ and $\theta$, respectively. Then it is known that

$$
-b_{n} \Delta_{\theta} u+R u=\widetilde{R} u^{p-1}
$$

where

$$
b_{n}=\frac{2(2 n+1)}{n+1}, \quad p=2+\frac{2}{n},
$$

and $\Delta_{\theta}$ is the sublaplacian for $\theta$. Equation (3-2) is called the CR Yamabe equation. The $C R$ Yamabe problem is to find a positive solution of (3-2) that makes $\widetilde{R}$ constant. One may refer to [Jerison and Lee 1987; 1989; Lee 1986] for the properties of the CR Yamabe equation and the solvability of the CR Yamabe problem.

Now let us consider the situation of Theorem 1.4. Let $M$ be a smooth real hypersurface in $\mathbb{C}^{n+1}, p \in M$ be a strongly pseudoconvex point and let $f: M \rightarrow M$ be a CR mapping satisfying that $f(p)=p, d f_{p}=\operatorname{Id}_{T_{p} M}$ and that the iteration sequence $\left\{f^{k}: k \in \mathbb{Z}_{+}\right\}$is compact. Let $\Gamma$ be the connected component of the set of strongly pseudoconvex points in $M$ that contains $p$. One should notice that Theorem 2.1 is still valid for subelliptic cases, since the proof depends only on the Fredholm alternative theorem. Therefore, by a similar argument as in the conformal case, we can choose a neighborhood $U$ of $p$ in $\Gamma$ and a contact 1-form $\theta$ on $\Gamma$ such that

- $f$ is one-to-one, and

- the Webster scalar curvature for $\theta$ vanishes on $U$.

Take a relatively compact neighborhood $V$ of $p$ in $U$ such that $f^{k}(V) \subset U$ for every $k=1,2, \ldots$. Then an iteration argument as in the conformal case and the subellipticity of the sublaplacian yield that $u-1$ vanishes at $p$ up to infinite order, where $u$ is the positive function on $V$ defined by

$$
f^{*} \theta=u^{2 / n} \theta \text {. }
$$

Remark 3.1. Although $\Delta_{\theta} u=0$ on $V$, we cannot conclude that $u \equiv 1$ at this stage, since the unique continuation principle for subelliptic operator has not been completely solved. In fact, an example in [Bahouri 1986] shows that a continuation theorem of Garofalo-Lin-Kazdan type cannot hold in the 3-dimensional Heisenberg group. Some partial results on the unique continuation principle for the sublaplacian were obtained in [Garofalo and Lanconelli 1990] for the Heisenberg group and in [Niu and Wang 2010] for more general nilpotent groups. 
Let $g$ be the Riemannian metric on $\Gamma$ defined by (3-1). Then (3-3) yields that

$$
\tilde{g}:=f^{*} g=\lambda^{2} \theta \otimes \theta+\lambda L_{\theta},
$$

where $\lambda=u^{2 / n}$. Since $u-1$ vanishes at $p$ up to infinite order, so does $\lambda-1$.

Let $\left(x^{0}, \ldots, x^{2 n}\right)$ be local coordinates centered at $p$ such that $g_{i j}(0)=\delta_{i j}$. Since $\lambda-1$ vanishes at $p=0$ up to infinite order, the Taylor coefficients of $g_{i j}$ and $\tilde{g}_{i j}$ at $p=0$ coincide. Since derivatives of $f$ at 0 of order $\geq 2$ are completely determined by differences between the Taylor coefficients of $g_{i j}$ and $\tilde{g}_{i j}$, we see that $f$ coincides with the identity at 0 up to infinite order. Note that $f$ is a CR diffeomorphism from $V$ onto $f(V)$. Since every local CR diffeomorphism on a strongly pseudoconvex CR manifold is uniquely determined by its finite order jet at the fixed point [Chern and Moser 1974; Kim and Zaitsev 2005], we conclude that $f \equiv \operatorname{Id}$ on $V$.

If $M$ is a compact real hypersurface that bounds a domain $D$, then the CR mapping $f$ extends continuously to a holomorphic map $F$ on $D$ by the Bochner-Hartogs extension theorem. Since $F-\operatorname{Id}_{D}$ vanishes on an open piece $V$ of the boundary $\partial D=M$, we see that $F$ and hence $f$ coincide with the identity map. Now suppose that $M$ is a real hypersurface in $\mathbb{C}^{n+1}$ containing no analytic hypersurface. Let

$$
F=\{x \in M: f(x)=x\}
$$

and let $F^{\circ}$ be the interior of $F$, which is nonempty by the argument above. Let $x_{0}$ be a limit point of $F^{\circ}$. By Trépreau's theorem [1986], there exists a neighborhood $\Omega$ of $x_{0}$ in $\mathbb{C}^{n+1}$ such that $\Omega \backslash M=\Omega_{+} \cup \Omega_{-}$and such that $f$ extends continuously to a holomorphic map $F$ defined on $\Omega_{+}$. Since $F \equiv$ Id on a nonempty open piece $F^{\circ} \cap \Omega$ in $\partial \Omega_{+}$, we see that $F \equiv \mathrm{Id}$ on $\Omega_{+}$. Therefore, $x_{0} \in F^{\circ}$. This yields the conclusion.

Corollary 3.2. Let $M$ be a real hypersurface in $\mathbb{C}^{n+1}$ containing no analytic hypersurface. Let $p$ be a strongly pseudoconvex point of $M$. If $f: M \rightarrow M$ is a $C R$ automorphism that $f(p)=p$ and $d f_{p}=\mathrm{Id}$, then either $f=\operatorname{Id}$ or $M$ is CR equivalent to the sphere $S^{2 n+1}$, and $f$ can be transformed into the CR transformation $\phi_{a, r}$ of $S^{2 n+1}$ fixing $p_{0}=(1,0, \ldots, 0) \in \mathbb{C}^{n+1}$ for some $a=\left(a_{1}, \ldots, a_{n}\right) \in \mathbb{C}^{n}$ and $r \in \mathbb{R}$, where $\phi_{a, r}=\Phi_{a, r} / \Psi_{a, r}$ and $\Phi_{a, r}$ is the affine transformation of $\mathbb{C}^{n+1}$ defined by

$$
\begin{aligned}
& w^{0}=\left(1-\frac{1}{2}\left(|a|^{2}+i r\right)\right) z^{0}-i \sum_{j=1}^{n} \bar{a}^{j} z^{j}+\frac{1}{2}\left(|a|^{2}+i r\right), \\
& w^{j}=z^{j}+i a^{j}\left(1-z^{0}\right)
\end{aligned}
$$

for $j=1, \ldots, n$, and $\Psi_{a, r}$ is the $\mathbb{C}$-valued function defined by

$$
\Psi_{a, r}(z)=\frac{1}{2}\left(|a|^{2}+2+i r\right)-i \sum_{j=1}^{n} \bar{a}^{j} z^{j}-\frac{1}{2}\left(|a|^{2}+i r\right) z^{0} .
$$


Proof. By Theorem 1.4, we may assume that $\left\{f^{k}: k \in \mathbb{Z}\right\}$ is noncompact if $f$ is not the identity map. Let $\Gamma$ be the connected component of the set of strongly pseudoconvex points that contains $p$. Since $f$ is a CR diffeomorphism of $M$ onto $M$, it preserves $\Gamma$. Moreover, the group $\left\{f_{\mid \Gamma}^{k}\right\}$ is also noncompact, since otherwise, $f_{\mid \Gamma} \equiv$ Id by Theorem 1.4 and this implies that $f \equiv$ Id on $M$. Therefore, Schoen's theorem [1995] implies that $\Gamma$ is CR equivalent to either the Heisenberg group $\mathbf{H}^{n}$ or the standard unit sphere in $\mathbb{C}^{n+1}$. Since $S^{2 n+1}$ is the one point compactification of $\mathbf{H}^{n}$, we also may assume that $f$ is a CR transformation of $S^{2 n+1}$ fixing $p_{0}=(1,0, \ldots, 0)$. By a result in [Webster 1977], either $f$ has two fixed points or it has only one fixed point. If $f$ has two fixed points, then each fixed point is a contracting fixed point of $f$ or $f^{-1}$. This contradicts the hypothesis that $d f_{p_{0}}=$ Id. Hence $f$ has only one fixed point $p_{0}$. In particular, $\Gamma$ cannot be equivalent to the Heisenberg group. Since $S^{2 n+1}$ is a boundary-free compact manifold, we can conclude that $M=\Gamma$ and that $M$ is CR equivalent to the unit sphere $S^{2 n+1}$.

To obtain explicit formulas for CR transformations of $S^{2 n+1}$ it is useful to imbed $S^{2 n+1}$ into the complex projective $(n+1)$-space $\mathbb{C} \mathrm{P}^{n+1}$ in the following manner: Let $\mathbb{C}^{n+1}$ be a complex Euclidean $(n+1)$-space with a coordinate system $\left(z^{0}, \ldots, z^{n}\right)$, and let $S^{2 n+1}$ be given by the equation

$$
\left|z^{0}\right|^{2}+\left|z^{1}\right|^{2}+\cdots+\left|z^{n}\right|^{2}=1 .
$$

We also let $\mathbb{C}^{n+2}$ be a complex Euclidean $(n+2)$-space with a coordinate system $\left(Z^{0}, Z^{1}, \ldots, Z^{n+1}\right)$, and let $\mathbb{C} \mathrm{P}^{n+1}$ be the projective $(n+1)$-space with a homogeneous coordinate system $\left[Z^{0}, Z^{1}, \ldots, Z^{n+1}\right]$. We define a holomorphic embedding of $\mathbb{C}^{n+1}$ into $\mathbb{C} \mathrm{P}^{n+1}$ by the equations

$$
Z^{0}=1+z^{0}, \quad Z^{j}=z^{j}(j=1, \ldots, n), \quad Z^{n+1}=i\left(1-z^{0}\right),
$$

and the image of $S^{2 n+1}$ in $\mathbb{C} \mathrm{P}^{n+1}$ under this embedding is the real hypersurface $Q$ that is defined by

$$
\left|Z^{1}\right|^{2}+\cdots+\left|Z^{n}\right|^{2}+\frac{i}{2}\left(Z^{n+1} \bar{Z}^{0}-Z^{0} \bar{Z}^{n+1}\right)=0 .
$$

The special unitary group $\mathrm{SU}(n+1,1)$ is the group of the linear transformations of $\mathbb{C}^{n+2}$ leaving the quadratic form

$$
\left|Z^{1}\right|^{2}+\cdots+\left|Z^{n}\right|^{2}+\frac{i}{2}\left(Z^{n+1} \bar{Z}^{0}-Z^{0} \bar{Z}^{n+1}\right)
$$

invariant, and whose determinant has absolute value 1 . We can regard the CR transformation group of $S^{2 n+1}$ as $\mathrm{SU}(n+1,1)$. The Lie algebra of $\mathrm{SU}(n+1,1)$ 
consists of $(n+2) \times(n+2)$ matrices of the form

$$
\left(\begin{array}{ccc}
\lambda & -2 i^{t} \bar{a} & r \\
-\frac{1}{2 i} \bar{b} & B & a \\
q & { }^{t} b & -\bar{\lambda}
\end{array}\right),
$$

where $B$ is a skew-hermitian $n \times n$ matrix, $a$ and $b$ are column $n$-vectors with complex entries, and $r, q$ are real numbers.

In particular, the Lie algebra of the isotropy group $\mathrm{SU}_{p_{0}}(n+1,1)$ at the point $p_{0}$ consists of the matrices of the form

$$
\left(\begin{array}{ccc}
\lambda & -2 i^{t} \bar{a} & r \\
0 & B & a \\
0 & 0 & -\bar{\lambda}
\end{array}\right)
$$

If $\lambda$ is not purely imaginary, then $f$ has two fixed points, by [Webster 1977]. This contradicts our hypothesis. Write $\lambda=i \theta$ for some $\theta \in \mathbb{R}$. The isotropy group $\mathrm{SU}_{p_{0}}(n+1,1)$ itself, as a subgroup of $\mathrm{SU}(n+1,1)$, consists of the matrices of the form

$$
\left(\begin{array}{ccc}
e^{i \theta} & -2 i e^{i \theta}\left({ }^{t} \bar{a}\right) & -i e^{i \theta}|a|^{2}+r e^{i \theta} \\
0 & T & T a \\
0 & 0 & e^{i \theta}
\end{array}\right),
$$

where $T \in \mathrm{SU}(n)$ and $r$ is a real number. Hence we can consider $f$ as a linear transformation of the form (3-5). Since $\left.d f_{p_{0}}\right|_{T_{p_{0}} S^{2 n+1}}=\mathrm{Id}, T$ is the identity map. So $f$ is represented by the matrix

$$
\left(\begin{array}{ccc}
e^{i \theta} & -2 i e^{i \theta}\left({ }^{t} \bar{a}\right) & -i e^{i \theta}|a|^{2}+r e^{i \theta} \\
0 & \operatorname{Id} & a \\
0 & 0 & e^{i \theta}
\end{array}\right) .
$$

By Webster [1977], the CR transformation represented by (3-6) has only one fixed point.

Since $\left[Z^{0}, \ldots, Z^{n+1}\right]$ is the homogeneous coordinate of $\mathbb{C P} \mathrm{P}^{n+1}$, the inverse map of (3-4) is given by

$$
z^{0}=\frac{i Z^{0}-Z^{n+1}}{i Z^{0}+Z^{n+1}} \quad \text { and } \quad z^{j}=\frac{2 i Z^{j}}{i Z^{0}+Z^{n+1}}
$$

for $j=1, \ldots, n$. If $e^{i \theta}$ is not 1 , then the map represented by the matrix (3-6) does not satisfy that $\left.d f_{p_{0}}\right|_{T_{p_{0}} S^{2 n+1}}=\mathrm{Id}$. This implies that $e^{i \theta}=1$. Using (3-6) and (3-7) we can conclude that $f=\Phi_{a, r} / \Psi_{a, r}$ for some $a \in \mathbb{C}^{n}$ and $r \in \mathbb{R}$. 


\section{References}

[Aubin 1976a] T. Aubin, "Équations différentielles non linéaires et problème de Yamabe concernant la courbure scalaire”, J. Math. Pures Appl. (9) 55:3 (1976), 269-296. MR 55 \#4288 Zbl 0336.53033 [Aubin 1976b] T. Aubin, "Problèmes isopérimétriques et espaces de Sobolev", J. Differential Geom. 11:4 (1976), 573-598. MR 56 \#6711 Zbl 0371.46011

[Bahouri 1986] H. Bahouri, "Non prolongement unique des solutions d'opérateurs "somme de carrés"”, Ann. Inst. Fourier (Grenoble) 36:4 (1986), 137-155. MR 88c:35027 Zbl 0603.35008

[Bell 1987] S. Bell, "Compactness of families of holomorphic mappings up to the boundary", pp. 29-42 in Complex analysis (University Park, PA, 1986), edited by S. G. Krantz, Lecture Notes in Math. 1268, Springer, Berlin, 1987. MR 88k:32066 Zbl 0633.32020

[Chern and Moser 1974] S. S. Chern and J. K. Moser, "Real hypersurfaces in complex manifolds", Acta Math. 133 (1974), 219-271. MR 54 \#13112 Zbl 0302.32015

[Fischer-Colbrie and Schoen 1980] D. Fischer-Colbrie and R. Schoen, "The structure of complete stable minimal surfaces in 3-manifolds of nonnegative scalar curvature", Comm. Pure Appl. Math. 33:2 (1980), 199-211. MR 81i:53044 Zbl 0439.53060

[Garofalo and Lanconelli 1990] N. Garofalo and E. Lanconelli, "Frequency functions on the Heisenberg group, the uncertainty principle and unique continuation", Ann. Inst. Fourier (Grenoble) 40:2 (1990), 313-356. MR 91i:22014 Zbl 0694.22003

[Garofalo and Lin 1987] N. Garofalo and F.-H. Lin, "Unique continuation for elliptic operators: a geometric-variational approach", Comm. Pure Appl. Math. 40:3 (1987), 347-366. MR 88j:35046 Zbl 0674.35007

[Gilbarg and Trudinger 1983] D. Gilbarg and N. S. Trudinger, Elliptic partial differential equations of second order, 2nd ed., Grundlehren der Mathematischen Wissenschaften 224, Springer, Berlin, 1983. MR 86c:35035 Zbl 0562.35001

[Huang 1993] X. J. Huang, "Some applications of Bell's theorem to weakly pseudoconvex domains", Pacific J. Math. 158:2 (1993), 305-315. MR 93m:32032 Zbl 0807.32016

[Jerison and Lee 1987] D. Jerison and J. M. Lee, "The Yamabe problem on CR manifolds", J. Differential Geom. 25:2 (1987), 167-197. MR 88i:58162 Zbl 0661.32026

[Jerison and Lee 1989] D. Jerison and J. M. Lee, "Intrinsic CR normal coordinates and the CR Yamabe problem”, J. Differential Geom. 29:2 (1989), 303-343. MR 90h:58083 Zbl 0671.32016

[Kazdan 1988] J. L. Kazdan, "Unique continuation in geometry", Comm. Pure Appl. Math. 41:5 (1988), 667-681. MR 89k:35039 Zbl 0632.35015

[Kim and Zaitsev 2005] S.-Y. Kim and D. Zaitsev, "Equivalence and embedding problems for CRstructures of any codimension”, Topology 44:3 (2005), 557-584. MR 2005j:32037 Zbl 1079.32022

[Krantz 1987] S. G. Krantz, "A new compactness principle in complex analysis", Universidad Autonoma de Madrid Seminarios 3 (1987), 171-194.

[Lee 1986] J. M. Lee, "The Fefferman metric and pseudo-Hermitian invariants", Trans. Amer. Math. Soc. 296:1 (1986), 411-429. MR 87j:32063 Zbl 0595.32026

[Niu and Wang 2010] P. Niu and J. Wang, "On unique continuation properties for the sub-Laplacian on Carnot groups", Acta Math. Sci. Ser. B Engl. Ed. 30:5 (2010), 1776-1784. MR 2012c:35076 Zbl 05951858

[Obata 1970] M. Obata, "Conformal transformations of Riemannian manifolds", J. Differential Geom. 4 (1970), 311-333. MR 42 \#2387 Zbl 0205.52003

[Schoen 1984] R. Schoen, "Conformal deformation of a Riemannian metric to constant scalar curvature”, J. Differential Geom. 20:2 (1984), 479-495. MR 86i:58137 Zbl 0576.53028 
[Schoen 1995] R. Schoen, "On the conformal and CR automorphism groups", Geom. Funct. Anal. 5:2 (1995), 464-481. MR 96h:53047 Zbl 0835.53015

[Tanaka 1975] N. Tanaka, A differential geometric study on strongly pseudo-convex manifolds, Lectures in Mathematics 9, Kinokuniya, Tokyo, 1975. MR 53 \#3361 Zbl 0331.53025

[Trépreau 1986] J.-M. Trépreau, "Sur le prolongement holomorphe des fonctions C-R défines sur une hypersurface réelle de classe $C^{2}$ dans $\mathbb{C}^{n}$ ", Invent. Math. 83:3 (1986), 583-592. MR 87f:32035 Zbl 0586.32016

[Trudinger 1968] N. S. Trudinger, "Remarks concerning the conformal deformation of Riemannian structures on compact manifolds", Ann. Scuola Norm. Sup. Pisa (3) 22 (1968), 265-274. MR 39 \#2093 Zbl 0159.23801

[Webster 1977] S. M. Webster, "On the transformation group of a real hypersurface", Trans. Amer. Math. Soc. 231:1 (1977), 179-190. MR 58 \#1231 Zbl 0368.57013

[Webster 1978] S. M. Webster, "Pseudo-Hermitian structures on a real hypersurface", J. Differential Geom. 13:1 (1978), 25-41. MR 80e:32015 Zbl 0379.53016

[Yamabe 1960] H. Yamabe, "On a deformation of Riemannian structures on compact manifolds", Osaka Math. J. 12 (1960), 21-37. MR 23 \#A2847 Zbl 0096.37201

Received November 8, 2011.

YOUNG-JUN CHOI

SCHOOL OF MATHEMATICS

Korea Institute For AdVEnCED STUdy

85 HOEGIRO

DONGDAEMON-GU

SEOUL 130-722

SOUTH KOREA

choiyj@kias.re.kr

JAE-CHEON JOO

DEPARTMENT OF MATHEMATICS

PUSAN NATIONAL UniVERSITY

GEUMJEONG-GU

BUSAN 609-735

SOUTH KOREA

jcjoo91@pusan.ac.kr 


\title{
PACIFIC JOURNAL OF MATHEMATICS
}

\author{
http://pacificmath.org \\ Founded in 1951 by \\ E. F. Beckenbach (1906-1982) and F. Wolf (1904-1989)
}

\section{EDITORS}

V. S. Varadarajan (Managing Editor)

Department of Mathematics

University of California

Los Angeles, CA 90095-1555

pacific@math.ucla.edu

Vyjayanthi Chari

Department of Mathematics

University of California

Riverside, CA 92521-0135

chari@math.ucr.edu

\section{Robert Finn}

Department of Mathematics Stanford University

Stanford, CA 94305-2125

finn@math.stanford.edu

Kefeng Liu

Department of Mathematics

University of California

Los Angeles, CA 90095-1555

liu@math.ucla.edu
Darren Long

Department of Mathematics

University of California

Santa Barbara, CA 93106-3080

long@math.ucsb.edu

Jiang-Hua Lu

Department of Mathematics

The University of Hong Kong

Pokfulam Rd., Hong Kong jhlu@maths.hku.hk

Alexander Merkurjev

Department of Mathematics

University of California

Los Angeles, CA 90095-1555

merkurev@math.ucla.edu
Sorin Popa

Department of Mathematics University of California

Los Angeles, CA 90095-1555 popa@math.ucla.edu

Jie Qing

Department of Mathematics

University of California

Santa Cruz, CA 95064

qing@cats.ucsc.edu

Jonathan Rogawski

Department of Mathematics

University of California

Los Angeles, CA 90095-1555

jonr@math.ucla.edu

\section{PRODUCTION}

pacific@math.berkeley.edu

\section{SUPPORTING INSTITUTIONS}

ACADEMIA SINICA, TAIPEI

CALIFORNIA INST. OF TECHNOLOGY INST. DE MATEMÁTICA PURA E APLICADA KEIO UNIVERSITY

MATH. SCIENCES RESEARCH INSTITUTE NEW MEXICO STATE UNIV.

OREGON STATE UNIV.

\author{
STANFORD UNIVERSITY \\ UNIV. OF BRITISH COLUMBIA \\ UNIV. OF CALIFORNIA, BERKELEY \\ UNIV. OF CALIFORNIA, DAVIS \\ UNIV. OF CALIFORNIA, LOS ANGELES \\ UNIV. OF CALIFORNIA, RIVERSIDE \\ UNIV. OF CALIFORNIA, SAN DIEGO \\ UNIV. OF CALIF., SANTA BARBARA
}

\author{
UNIV. OF CALIF., SANTA CRUZ \\ UNIV. OF MONTANA \\ UNIV. OF OREGON \\ UNIV. OF SOUTHERN CALIFORNIA \\ UNIV. OF UTAH \\ UNIV. OF WASHINGTON \\ WASHINGTON STATE UNIVERSITY
}

These supporting institutions contribute to the cost of publication of this Journal, but they are not owners or publishers and have no responsibility for its contents or policies.

See inside back cover or pacificmath.org for submission instructions.

The subscription price for 2012 is US \$420/year for the electronic version, and \$485/year for print and electronic.

Subscriptions, requests for back issues from the last three years and changes of subscribers address should be sent to Pacific Journal of Mathematics, P.O. Box 4163, Berkeley, CA 94704-0163, U.S.A. Prior back issues are obtainable from Periodicals Service Company, 11 Main Street, Germantown, NY 12526-5635. The Pacific Journal of Mathematics is indexed by Mathematical Reviews, Zentralblatt MATH, PASCAL CNRS Index, Referativnyi Zhurnal, Current Mathematical Publications and the Science Citation Index.

The Pacific Journal of Mathematics (ISSN 0030-8730) at the University of California, c/o Department of Mathematics, 969 Evans Hall, Berkeley, CA 94720-3840, is published monthly except July and August. Periodical rate postage paid at Berkeley, CA 94704, and additional mailing offices. POSTMASTER: send address changes to Pacific Journal of Mathematics, P.O. Box 4163, Berkeley, CA 94704-0163.

PJM peer review and production are managed by EditFLOW ${ }^{\mathrm{TM}}$ from Mathematical Sciences Publishers.

PUBLISHED BY PACIFIC JOURNAL OF MATHEMATICS

at the University of California, Berkeley 94720-3840

A NON-PROFIT CORPORATION

Typeset in LATEX

Copyright $(02012$ by Pacific Journal of Mathematics 


\section{PACIFIC JOURNAL OF MATHEMATICS}

Volume $258 \quad$ No. $2 \quad$ August 2012

Uniqueness theorems for $\mathrm{CR}$ and conformal mappings

YounG-JUn CHOI and JAE-CHEON JOO

Some finite properties for vertex operator superalgebras

269

CHONGYING DONG and JIANZHI HAN

On the geometric flows solving Kählerian inverse $\sigma_{k}$ equations

291

HAO FANG and MIJIA LAI

An optimal anisotropic Poincaré inequality for convex domains

GUOFANG WANG and CHAO XIA

Einstein metrics and exotic smooth structures

MASASHI ISHIDA

Noether's problem for $\hat{S}_{4}$ and $\hat{S}_{5}$

MING-CHANG KANG and JiAn ZHOU

Remarks on the behavior of nonparametric capillary surfaces at corners 369

KIRK E. LANCASTER

Generalized normal rulings and invariants of Legendrian solid torus links

MIKHAIL LAVROV and DAN RUTHERFORD

Classification of singular $\mathbb{Q}$-homology planes II: $\mathbb{C}^{1}$ - and $\mathbb{C}^{*}$-rulings.

KAROL PALKA

A dynamical interpretation of the profile curve of CMC twizzler surfaces

OSCAR M. PERDOMO

Classification of Ising vectors in the vertex operator algebra $V_{L}^{+}$

HIROKI SHIMAKURA

Highest-weight vectors for the adjoint action of $\mathrm{GL}_{n}$ on polynomials 